\title{
Iron acquisition with the natural siderophore enantiomers pyochelin and enantio-pyochelin in Pseudomonas species
}

\author{
Zeb A. Youard · Nicolas Wenner • \\ Cornelia Reimmann
}

Received: 16 November 2010/Accepted: 13 December 2010/Published online: 25 December 2010

(C) Springer Science+Business Media, LLC. 2010

\begin{abstract}
The bacterial siderophore pyochelin is composed of salicylate and two cysteine-derived heterocycles, the second of which is modified by reduction and $\mathrm{N}$-methylation during biosynthesis. In Pseudomonas aeruginosa, the first cysteine residue is converted to its $\mathrm{D}$-isoform during thiazoline ring formation, whereas the second cysteine remains in its L-configuration. Stereochemistry is opposite in the Pseudomonas fluorescens siderophore enantio-pyochelin, in which the first ring originates from L-cysteine and the second ring from D-cysteine. Both siderophores promote growth of the producer organism during iron limitation and induce the expression of their biosynthesis genes by activating the transcriptional AraC-type regulator PchR. However, neither siderophore is functional as an iron carrier or as a transcriptional inducer in the other species, demonstrating that both processes are highly stereospecific. Stereospecificity of pyochelin/enantio-pyochelinmediated iron uptake is ensured at two levels: (i) by the outer membrane siderophore receptors and (ii) by the cytosolic PchR regulators.
\end{abstract}

Z. A. Youard · N. Wenner · C. Reimmann $(\bowtie)$ Département de Microbiologie Fondamentale, Université de Lausanne, Bâtiment Biophore, 1015 Lausanne, Switzerland

e-mail: Cornelia.Reimmann@unil.ch
Keywords Siderophore - Pseudomonas . Enantiomer · Pyochelin · Enantio-pyochelin . Stereospecificity

\section{Introduction}

Iron is a cofactor of many redox-dependent enzymes and therefore essential for most living organisms including bacteria. Despite its abundance, iron is not freely available to microorganisms under aerobic conditions, as it forms poorly soluble ferric hydroxides in the environment and is bound to transport and storage proteins in host organisms. To acquire iron, bacteria have developed sophisticated strategies involving the production of iron chelators termed siderophores. Released to the environment, siderophores bind iron with high affinity and transport it to the bacterial cytoplasm via specific outer membrane receptors (Guerinot 1994; Andrews et al. 2003; Wandersman and Delepelaire 2004). The Gram-negative bacterium Pseudomonas aeruginosa produces two siderophores, pyoverdine and pyochelin (Pch), and, in addition, utilizes a large number of xenosiderophores for iron uptake (Bodilis and Cornelis 2009; Cornelis 2010), a mechanism known as siderophore piracy. Structurally related pyoverdines are produced by all fluorescent pseudomonads and, due to their high affinity for iron, pyoverdines are regarded as primary 
siderophores of these bacteria (Cornelis 2010). In contrast, the affinity for iron is lower in pyochelin and other so-called secondary Pseudomonas siderophores, which, in addition to their role as iron carriers, often have other biological activities (Cornelis 2010).

We recently reported that the secondary siderophore of some Pseudomonas fluorescens strains is not Pch as expected from earlier studies (Castignetti 1997; Schmidli-Sacherer et al. 1997; Terano et al. 2002), but is in fact its optical antipode, which we have termed enantio-pyochelin (E-Pch; Youard et al. 2007). Interestingly, Pch and E-Pch are biologically active only in their producer species (Youard et al. 2007; Hoegy et al. 2009; Youard and Reimmann 2010), suggesting that iron uptake with these compounds is highly stereospecific.

Here we review the chemical structure, metal chelation and biosynthetic pathway of Pch and present our current knowledge on its enantiomer E-Pch. We compare the role of the two siderophores in iron uptake and transcriptional regulation in both
Pseudomonas species and discuss the importance and biological implications of chirality in these processes.

\section{The siderophores pyochelin and enantio-pyochelin}

Pch was isolated in the late 1970s from culture broth of Pseudomonas aeruginosa ATCC15692 (strain PAO1) grown under iron limitation, and its chemical structure was determined as 2-(2-o-hydroxyphenyl2-thiazolin-4-yl)-3-methylthiazolidine-4-carboxylic acid (Cox and Graham 1979; Cox 1980; Cox et al. 1981; Liu and Shokrani 1978) (Fig. 1a). Pch is poorly soluble in water and has been reported to bind ferric iron with a stochiometry of 2:1 (Pch to $\mathrm{Fe}^{3+}$ ) (Visca et al. 1992; Tseng et al. 2006) and a stability constant of $2 \times 10^{5} \mathrm{M}^{-1}$, determined in ethanol (Cox and Graham 1979). Pch binds, albeit with lower affinities, also other metals such as $\mathrm{Ag}^{+}, \mathrm{Al}^{3+}, \mathrm{Cd}^{2+}, \mathrm{Co}^{2+}$, $\mathrm{Cr}^{2+}, \mathrm{Cu}^{2+}, \mathrm{Eu}^{3+}, \mathrm{Ga}^{3+}, \mathrm{Hg}^{2+}, \mathrm{Mn}^{2+}, \mathrm{Mo}^{6+}, \mathrm{Ni}^{2+}$, $\mathrm{Pb}^{2+}, \mathrm{Sn}^{2+}, \mathrm{Tb}^{3+}, \mathrm{Tl}^{+}$, and $\mathrm{Zn}^{2+}$. However, besides<smiles>C#CC1(C(=O)O)CSC(C(C)C)(C2(C(=O)O)CSC(c3ccccc3O)=N2)N1C</smiles><smiles>[B]c1ccccc1C1=NC([C@@H]2SC[C@@H](C(=O)O)N2C)CS1</smiles>

Pyochelin II $\left(4^{\prime} R, 2^{\prime \prime} S, 4^{\prime \prime} R\right)$

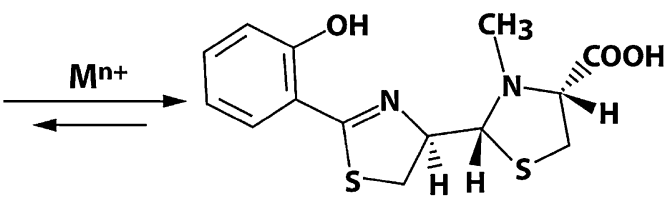

Pyochelin I (4'R, 2"R, 4"R)<smiles></smiles>

Fig. 1 Structural configurations of Pch and E-Pch isomers. $\mathrm{Pch}$ and E-Pch have three chiral carbons, $\mathrm{C}^{\prime}, \mathrm{C} 2^{\prime \prime}$, and $\mathrm{C}^{\prime \prime}$ (a) and exist in nature as pairs of interconvertible diastereoisomers. Pch I and II (b) are made by P. aeruginosa PAO1 (Ankenbauer et al. 1988; Rinehart et al. 1995), whereas E-Pch I and II (c) are produced by the P. fluorescens strains Pf-5 and CHA0 (Youard et al. 2007). Note that the metal $\left(\mathrm{M}^{\mathrm{n}+}\right)$-induced shift at C2" has only been shown for Pch (Schlegel et al. 2004). In ferriPch complexes iron is tetradentately coordinated by one Pch molecule (O1, N1, N2, O2) and bidentately by a second Pch molecule (O1, N1 or N2, O3) or any other available ligand (Cobessi et al. 2005; Klumpp et al. 2005; Hayen and Volmer 2006; Schlegel et al. 2006; Tseng et al. 2006) 
$\mathrm{Fe}^{3+}$, only $\mathrm{Co}^{2+}, \mathrm{Ga}^{3+}, \mathrm{Mo}^{6+}$ and $\mathrm{Ni}^{2+}$ were transported to some extent by Pch in $P$. aeruginosa (Braud et al. 2009; Namiranian et al. 1997; Visca et al. 1992).

In the 2:1 Pch-iron complex, four of the six octahedral coordination sites of ferric iron are occupied by the phenolate and the carboxylate oxygen ( $\mathrm{O} 1$ and $\mathrm{O} 3$, respectively) and by the two nitrogen atoms N1 and N2 of the first Pch molecule (Fig. 1a). The remaining two coordination sites can accomodate either $\mathrm{O} 1$ and $\mathrm{N} 1$ or $\mathrm{N} 2$ and $\mathrm{O} 3$ of the second Pch molecule (Tseng et al. 2006). However, Pch: $\mathrm{Fe}^{3+}$ (ferriPch) complexes with a 1:1 stoichiometry have been reported as well. In these complexes, the remaining two coordination sites of ferric iron were occupied by other available ligands (Klumpp et al. 2005; Cobessi et al. 2005; Hayen and Volmer 2006; Tseng et al. 2006; Schlegel et al. 2006). Thus, the nature of the biologically relevant ferriPch complex is not entirely clear and may depend on ligand concentrations in vivo.

Pch has three chiral centers $\left(\mathrm{C}^{\prime}, \mathrm{C} 2^{\prime \prime}\right.$, and $\mathrm{C}^{\prime \prime}$; Fig. 1a) and is extracted from P. aeruginosa PAO1 as a mixture of two interconvertible diastereoisomers whose absolute configuration was determined as $4^{\prime} R$, $2^{\prime \prime} R, 4^{\prime \prime} R$ (Pch I) and $4^{\prime} R, 2^{\prime \prime} S, 4^{\prime \prime} R$ (Pch II) (Fig. 1b; Ankenbauer et al. 1988; Rinehart et al. 1995). In the presence of $\mathrm{Fe}^{3+}$ or $\mathrm{Zn}^{2+}$, the $S$ configuration at the chiral center $\mathrm{C}^{\prime \prime}$ is converted to the $R$ configuration, inducing a shift from Pch II to the iron-binding diastereoisomer Pch I (Fig. 1b; Ino and Murabayashi 2001; Schlegel et al. 2004, 2006).

The two diastereoisomers isolated from the Pseudomonas fluorescens strains Pf-5 and CHA0 have a stereochemistry opposite to that of Pch I and II and were thus named E-Pch I $\left(4^{\prime} S, 2^{\prime \prime} S\right.$, and $\left.4^{\prime \prime} S\right)$ and E-Pch II $\left(4^{\prime} S, 2^{\prime \prime} R\right.$, and $4^{\prime \prime} S$ ) (Fig. 1c; Youard et al. 2007). By analogy with Pch, a metal-induced shift from E-Pch II to E-Pch I is expected to occur and it is assumed that E-PchI is the iron-binding diastereoisomer.

\section{Biosynthesis}

Pch and E-Pch are condensation products of salicylate and two molecules of cysteine, which are cyclized during the assembly and undergo a certain number of modifications (Fig. 2). The sole difference between Pch and E-Pch is the stereochemical configuration of the two cysteines incorporated. In Pch biosynthesis, it is the first L-cysteine that undergoes an epimerization reaction, while in E-Pch biosynthesis it is the second L-cysteine that is converted to its D-isoform. In the following we will briefly review the Pch biosynthesis pathway (Fig. 2a) and then highlight the differences and open questions with regard to E-Pch assembly (Fig. 2b).

The Pch biosynthesis genes of $P$. aeruginosa PAO1 are organized in two divergent operons, $p c h D C B A$ and pchEFGHI (Serino et al. 1997; Reimmann et al. 1998;
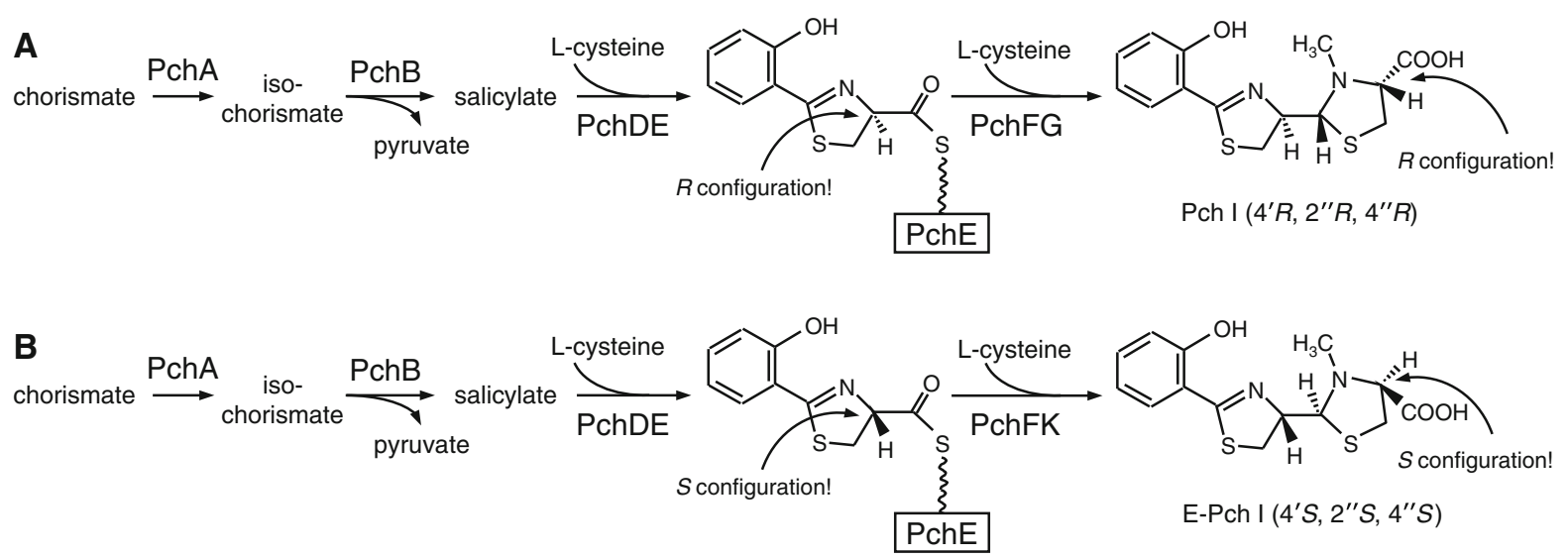

Fig. 2 Biosynthetic pathways generating Pch (a) in P. aeruginosa and E-Pch (b) in the P. fluorescens strains Pf-5 and CHA0. The Pch biosynthetic pathway has been studied with purified enzymes in vitro (see text for details) while the proposed pathway for E-Pch is essentially based on bioinformatic analysis. Note that the chiral center $\mathrm{C}_{2}{ }^{\prime \prime}$ isomerizes spontaneously (Fig. 1) but for simplicity reasons, only the isomers I of the two siderophores are shown here 


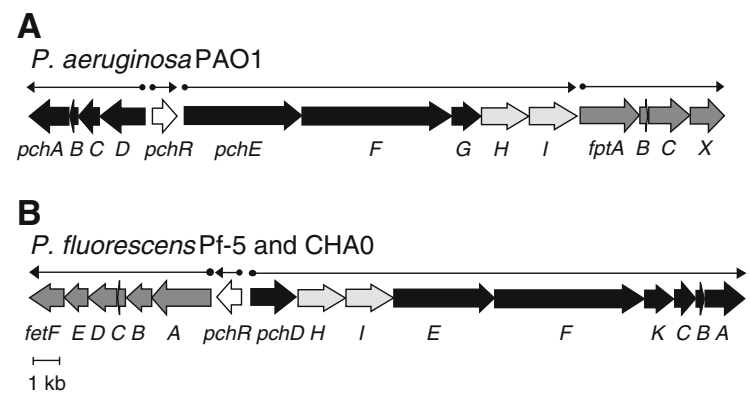

Fig. 3 Gene clusters for biosynthesis and transport of Pch in $P$. aeruginosa PAO1 (a) and for E-Pch in P. fluorescens Pf-5 and CHA0 (b), respectively. Genes for biosynthesis are in black, genes for siderophore uptake in dark grey, and the pchR regulatory genes are in white. The $\mathrm{ABC}$ transport genes pchHI, whose function in Pch transport is not clear, are shown in light grey. Transcriptional units (confirmed only for pchDCBA and fptABCX of $P$. aeruginosa [Serino et al. 1997; Michel et al. 2007]) are indicated by black arrows above the genes. The five genes located immediately downstream of the E-Pch receptor gene $f e t A$ (PFL_3498 in strain Pf-5) are named fetBCDEF here (PFL_3499 to PFL_3503 in strain Pf-5). Bioinformatics analysis predicts that FetCDE form a periplasmic binding protein-dependent $\mathrm{ABC}$ transporter potentially involved in $\mathrm{E}$ Pch transport across the inner membrane. The figure is based on sequence data from Stover et al. (2000), Paulsen et al. (2005), Youard et al. (2007), and Hoegy et al. (2009)

Reimmann et al. 2001), which are clustered with the pyochelin uptake operon $f p t A B C X$ (Ankenbauer and Quan 1994; Michel et al. 2007) and the regulatory gene pchR (Heinrichs and Poole 1993) (Fig. 3a). During the initial steps of Pch biosynthesis, chorismate is converted to salicylate via isochorismate (Fig. 2). These steps are carried out by the isochorismate synthase PchA (Serino et al. 1995; Gaille et al. 2003) and by the isochorismate pyruvate-lyase PchB (Serino et al. 1995; Gaille et al. 2002). The amino acid sequence of $\mathrm{PchB}$ is unrelated to pyruvate lyases of other shikimic acid metabolites, and its crystal structure reveals homology with chorismate mutases of the AroQ $\alpha$ class (Zaitseva et al. 2006). Indeed, PchB is a bifunctional enzyme displaying, in addition, a chorismate mutase activity (Gaille et al. 2002; Künzler et al. 2005; DeClue et al. 2005). Conversion of chorismate to salicylate is a two-step process in Pch biosynthesis. In contrast, in the biosyntheses of other salicylatederived siderophores such as yersiniabactin and mycobactin, salicylate is made from chorismate in a single step, which is carried out by Irp9 and MbtI, respectively (Pelludat et al. 2003; Kerbarh et al. 2005; Kerbarh et al. 2006; Harrison et al. 2006).
In the subsequent steps of Pch biosynthesis salicylate is linked to two molecules of L-cysteine by a thiotemplate mechanism involving the salicylate adenylating enzyme PchD, the two peptide synthetases PchE and PchF, and the reductase PchG (Serino et al. 1997; Reimmann et al. 1998, 2001; Quadri et al. 1999; Patel and Walsh 2001; Patel et al. 2003; Fig. 2a). Pch synthesis from salicylate and L-cysteine has been fully reconstituted with purified enzymes in vitro (Patel and Walsh 2001) and the individual reactions have been studied in detail (Quadri et al. 1999; Reimmann et al. 2001; Patel and Walsh 2001; Patel et al. 2003; for a recent review see also Thomas 2007). The main steps are briefly summarized here. The substrates salicylate and L-cysteine are adenylated by PchD and PchE, respectively, and loaded onto PchE via covalent thioester bonds that are provided by two posttranslationally added phosphopantheteinyl prostetic groups. The subsequent PchEcatalyzed condensation and cyclization reactions generate an enzyme-bound hydroxyphenyl-thiazoline intermediate. As shown in Fig. 2a, the chiral center $\mathrm{C} 4^{\prime}$ of this intermediate has an $R$ configuration, indicating that the thiazoline ring is derived from D-cysteine rather than L-cysteine. During ring formation, an epimerization reaction is carried out by a PchE-embedded epimerase domain, which converts the PchE-bound L-cysteinyl residue to its D-isoform (Reimmann et al. 1998; Patel et al. 2003). Meanwhile, a second L-cysteine molecule is adenylated by the peptide synthetase PchF and anchored, again via a phosphopantetheinyl linker, to a carrier domain in PchF. PchF then catalyzes the condensation of the PchE-bound hydroxyphenyl-thiazoline intermediate with L-cysteinyl-PchF and generates the second thiazoline ring (Quadri et al. 1999). The Pch backbone is now completed and the second thiazoline ring undergoes reduction and methylation before the final product is released from its thiotemplate. Reduction is carried out by the reductase PchG generating a thiazolidine ring. A methyltransferase domain in PchF then catalyzes N-methylation of this ring. The completed Pch is released from PchF by the protein's C-terminal thioesterase domain.

Three of the pch genes shown in Fig. $3 \mathrm{a}$ are not essential for the production of Pch. PchC encodes a type II thioesterase, which maximizes Pch production in $P$. aeruginosa, probably by removing wrongly charged substrates from the peptidyl carrier protein 
domains of PchE and PchF (Reimmann et al. 2004). The $p c h H$ and $p c h I$ genes encode ABC transporters, whose potential function in Pch transport may be redundant, as a deletion of these genes did not reduce the amount of Pch found in culture supernatants of P. aeruginosa (Reimmann et al. 2001).

The biosynthesis of E-Pch in P. fluorescens has not yet been studied biochemically, but is probably quite similar to the Pch pathway in $P$. aeruginosa. A closely related gene cluster is present in the chromosome of the P. fluorescens strains Pf-5 and CHA0, although the arrangement of the individual genes is different from that in P. aeruginosa and there is no gene with obvious sequence homology to $p c h G$ (Paulsen et al. 2005; Youard et al. 2007; Fig. 3b). Bioinformatics predict that reduction of the second thiazoline ring in E-Pch biosynthesis could be catalyzed by PchK, a putative thiazolinyl reductase encoded downstream of pchF in $P$. fluorescens (Fig. 3b; H.J. Imker and C.T Walsh, personal communication).

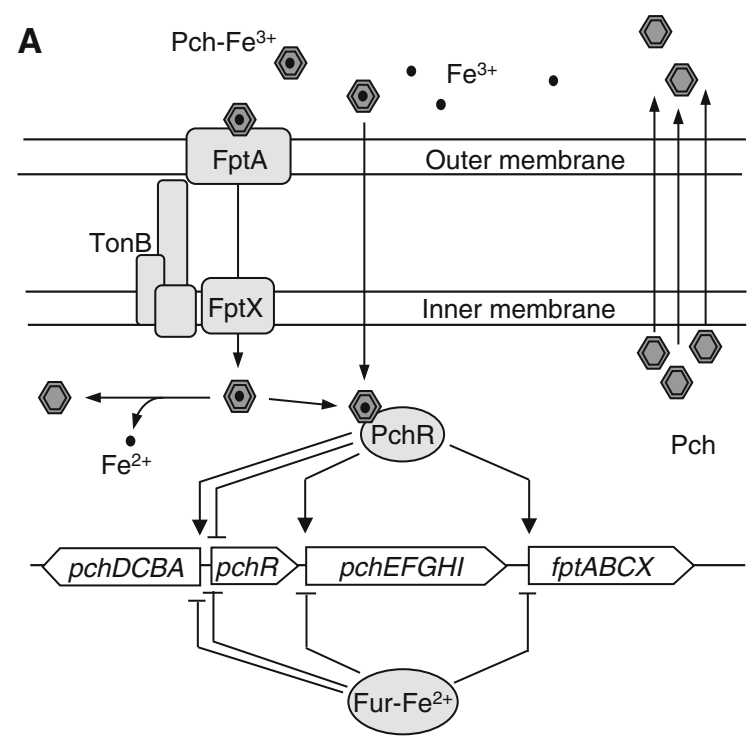

Fig. 4 Iron uptake with Pch and E-Pch and its transcriptional regulation. In $P$. aeruginosa (a), ferriPch crosses the outer membrane via the receptor FptA and the inner membrane via the permease FptX. FerriPch then activates the PchR regulator, which in turn induces the expression of pchDCBA, pchEFGHI, and fpt $A B C X$, and represses its own gene. Iron uptake and signalling with E-Pch in P. fluorescens (b) is expected to proceed in a similar way, except that after FetA-dependent transport through the outer membrane, the iron-siderophore complex may cross the inner membrane via a classical $\mathrm{ABC}$
How is the $S$ configuration at the chiral centers $\mathrm{C} 4^{\prime}$ and $\mathrm{C} 4^{\prime \prime}$ of E-Pch generated? Apparently, the $P$. fluorescens PchE does not have an epimerase domain (Youard et al. 2007). The first L-cysteine incorporated by this enzyme will therefore remain in its original L-conformation, generating an $S$ stereochemistry at $\mathrm{C}^{\prime}$ (Fig. 2b). The origin of the $S$ stereochemistry at $\mathrm{C}^{\prime \prime}$ in E-Pch is not known. When the $p c h D C B A_{\mathrm{PAO} 1}$ genes are co-expressed together with $p c h E F K_{\mathrm{CHA} 0}$, E-Pch is produced in $P$. aeruginosa, indicating that an epimerase function is encoded either by $p c h F_{\mathrm{CHA} 0}$ or by $p c h K_{\mathrm{CHAO}}$ (N. Wenner and C. Reimmann, unpublished results).

Iron uptake

The protein machineries transporting ferriPch and ferriE-Pch are not related. The ferriPch complex is recognized at the cell surface of $P$. aeruginosa and transported into the periplasm by a specific outer membrane transporter, FptA (Ankenbauer and Quan,

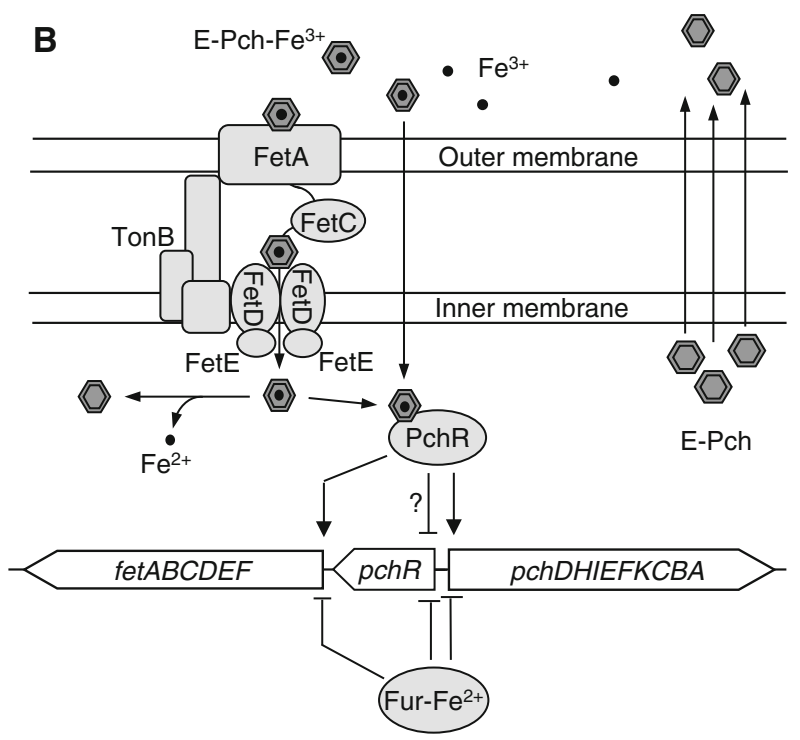

transport system encoded by genes of the fetABCDEF operon. Note that in the absence of FptA and FetA, respectively, activation of PchR by its cognate siderophore is reduced but not abolished (Youard and Reimmann 2010), implying that the siderophores can enter the cells also by an alternative, lowefficiency uptake pathway. Under iron-replete conditions, the Fur repressor blocks transcription of $p c h D C B A, p c h R$, pchEFGHI, and fptABCX in $P$. aeruginosa, and a similar repression of pchDHIEFKCBA, pchR, and fetABCDEF probably occurs in $P$. fluorescens (see text) 
1994; Fig. 4a). The structure of this protein (Cobessi et al. 2005) is typical for this class of transporters: a transmembrane $22-\beta$-stranded barrel occluded by an $\mathrm{N}$-terminal domain forming the cork. Binding of ferriPch to FptA occurs with high affinity $\left(K_{d}=2.5 \pm 1.1 \mathrm{nM}\right.$; Hoegy et al. 2009) and, consistent with the hydrophobicity of the siderophore, the ferriPch binding pocket is mainly composed of hydrophobic and aromatic residues. Once translocated to the periplasm, ferriPch crosses the inner membrane via FptX, a member of a novel family of permeases that function as single-subunit siderophore transporters (Ó Cuív et al. 2004; Fig. 4a). Iron release from Pch is expected to occur by a reductive process, although no experiments have been performed along these lines. The $f p t A$ and $f p t X$ genes form an operon together with $f p t B$ and $f p t C$ (Michel et al. 2007; Fig. 3a). The function of these latter genes is not clear, as their deletion does not affect the utilization of ferriPch as an iron source in P. aeruginosa (Michel et al. 2007).

Uptake of ferriE-Pch in $P$. fluorescens requires the FetA receptor (Youard et al. 2007; Fig. 4b) which binds ferriE-Pch with high affinity $\left(K_{d}=3.7 \pm 2.1\right.$ $\mathrm{nM}$ ) and functionally resembles FptA (Hoegy et al. 2009). FetA is encoded by the first gene of the putative E-Pch uptake operon fetABCDEF (Fig. 3b). Bioinformatics suggest that $f e t B$ encodes a PepSYassociated membrane protein whose function is not known. The fetCDE genes code for a classical ATPbinding cassette (ABC) transport system comprising a periplasmic binding protein (FetC), a permease (FetD), and an ATP-binding protein (FetE). We suspect that this $\mathrm{ABC}$ transporter is responsible for ferriE-Pch translocation across the inner membrane (Fig. 4b). The last gene of the operon, $f e t F$, encodes a transporter of the major facilitator superfamily (MFS). Could this transporter play a role in E-Pch recycling after iron release?

\section{Regulation}

Under iron-replete conditions, the biosynthesis of Pch and the uptake system for ferriPch are repressed by the ferric uptake regulator Fur, which, when complexed with ferrous iron, binds to a conserved sequence element (Fur-box) in the promoter regions of iron-regulated genes and blocks their transcription (Escolar et al. 1999). Fur-boxes are present in the promoters of the pchDCBA, pchEFGHI, pchR and fptABCX genes and expression studies have demonstrated Fur-dependent repression of these genes in iron-replete conditions (Heinrichs and Poole 1993; Ochsner et al. 1995; Ankenbauer and Quan 1994; Serino et al. 1997; Reimmann et al. 1998; Fig. 4a). When iron becomes limiting, Fur dissociates from the Fur-boxes allowing a basal level of gene expression to occur. Full expression of pchDCBA, pchEFGHI, and $f p t A B C X$ requires the transcriptional activator PchR (Heinrichs and Poole 1993, 1996; Reimmann et al. 1998). Pch (probably in its iron-loaded form) acts as an effector molecule of PchR and allows this regulator to bind a 32-bp sequence motif (PchR-box) in promoter regions of these genes (Fig. 4a; Michel et al. 2005). Binding of PchR $\sim$ Pch to the PchR-box in the $p c h D$ promoter also represses transcription of $p c h R$ itself, as this PchR binding site is located downstream of the pchR transcription start (Michel et al. 2005).

Regulation in $P$. fluorescens is probably similar (Fig. 4b). Well-conserved Fur-boxes are present in the promoter regions of pchDHIEFKCBA, pchR, and fetABCDEF (Paulsen et al. 2005), suggesting that these genes are repressed under iron replete conditions. Moreover, potential PchR-boxes are found upstream of fetABCDEF and in the pchR-pchDHIEFKCBA intergenic region (Michel et al. 2005; Paulsen et al. 2005). Indeed, pchDHIEFKCBA expression was shown to require PchR and E-Pch (Youard et al. 2007; Youard and Reimmann 2010). As the transcription start sites of pchR and pchDHIEFKCBA have not yet been determined, it cannot be predicted if binding of PchR $\sim$ E-Pch to the PchR-box upstream of $p c h D$ will result in concomitant repression of $p c h R$ (Fig. $4 \mathrm{~b}$ ).

\section{Stereospecificity}

Iron uptake and transcriptional regulation with Pch and E-Pch are stereospecific processes in both Pseudomonas species (Youard et al. 2007; Hoegy et al. 2009; Youard and Reimmann 2010). Stereospecificity of iron uptake firstly involves the siderophore receptors FptA and FetA which are not closely related (Fig. 5a) and have opposite binding enantioselectivities. Hoegy et al. (2009) have shown that the Pch receptor FptA does not bind and transport ferriE-Pch, while the E-Pch receptor FetA is unable to interact with ferriPch. Docking experiments using 
Fig. 5 Amino acid sequence alignment of the outer membrane receptors FptA and FetA (a) and of the two regulatory proteins $\operatorname{PchR}_{\mathrm{Pa}}(P$. aeruginosa) and $\mathrm{PchR}_{\mathrm{Pf}}$ (P. fluorescens)

(b) performed with ClustalW2. Note that FptA and FetA are not closely related $(25 \%$ identical amino acids) and that the highlighted residues F114, L116, L117, M271, Y334, Q395 and W702 which form the ferriPch binding pocket in FptA (Cobessi et al. 2005) are not conserved in the FetA receptor. In the PchR proteins, siderophore specificity maps to the $\mathrm{N}$-terminal part (amino acids $\sim 20$ to $\sim 170$; Youard and Reimmann 2010) while the C-terminal domain is involved in DNA binding (the two helix-turnhelix DNA binding motifs are highlighted by black bars above the sequence alignment)
A

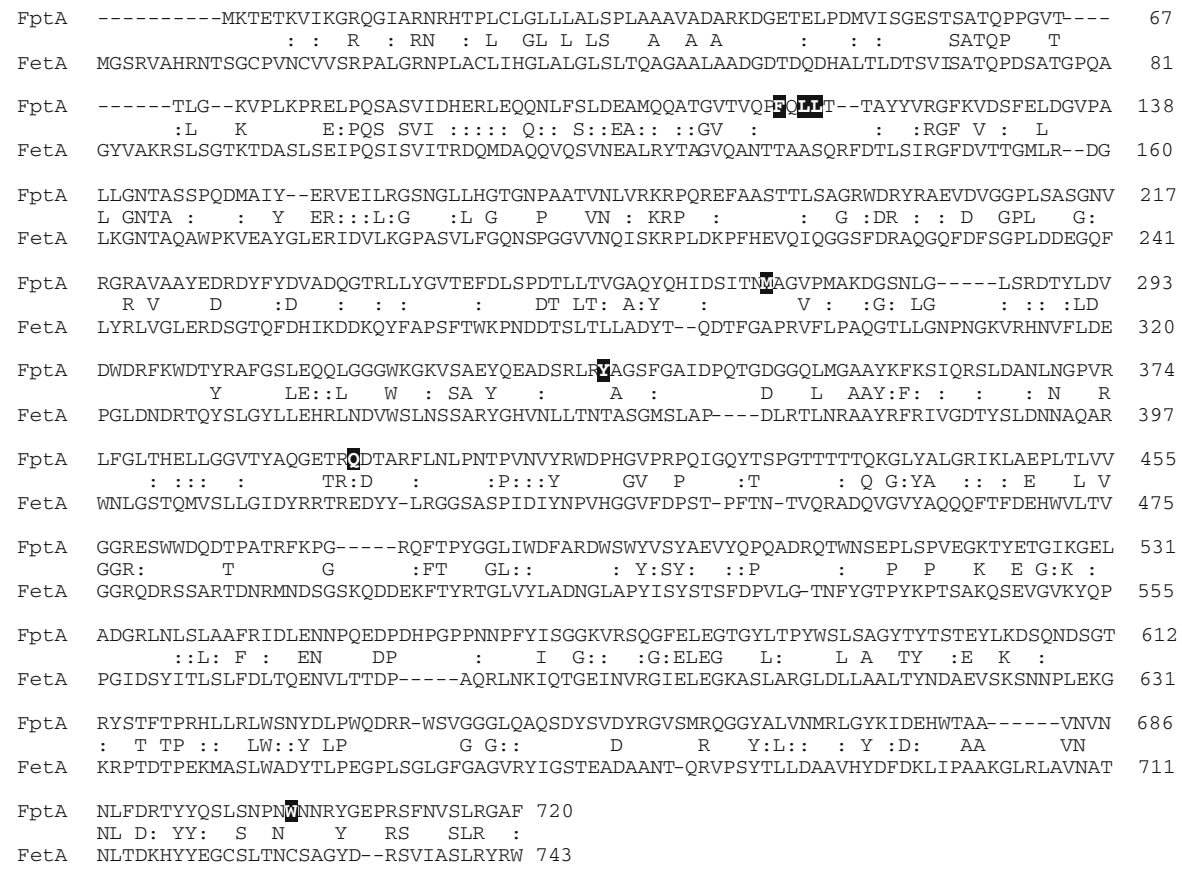

B

PChR $_{\mathrm{Pa}}$ MTITIIAPP--QADAAAPAPG-NRPGVAHIDPNMKLVTGTFCSASEDWFEEPLERGLRLILVQSGQLRCRIPGQPEHLIEG 78

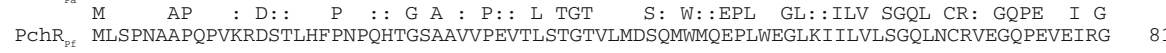

PChR $_{\mathrm{Pa}}$ PSLCTIANDGDFTSAQIYGTDKPLRYTIVQLGVEALDSRLGWLPEQLIRRPGGDPRIMSCPAPRAMQALASQIATCQMLGP 159 P:LC::AN:G: :: : : P:RYT VQL : : :G PE:L: : GG P :: PA : : ALA QI TC : GP $P_{\mathrm{PCh}}$ PTLCAVANQGEHCGDHLFASGVPVRYTVQLDFPSIRN-VGLEPERLLDQRGGGPMLFCQPASKPLLALAQQIFTCPLQGP 161

PChR $_{\text {pa }}$ TRDLYLGGKALELAALSAQ--FLSGEGRPVEEPRITCSEVERIHAARDLLVGALQEPPSLDTLASRVGMNPRKLTAGFRKV 238 TR :YLGGKALEL:AL : : E RP: : : : : ERIHAARDLL: :LO: PSL L: :VG:NPRKLTAGFR:V

PChR $_{\mathrm{Pf}}$ TRSFYLGGKALELTALGVEGILAQAESRPLQDSPCSSADIERIHAARDLLLNSLQDSPSLSELSRQVGLNPRKLTAGFRQV 242

PChR $_{\mathrm{pa}}$ FGASVFGYLEYRLREAHRMLCDEEANVSTVAYRVGYSPAHFSIAFRKRYGISPSEIR 296 FG:SV: YLQE RL A:R:L E:NVS: AYRVGYSPAHFS AFRKR:G:SP :R

$\begin{array}{lll}\text { PChR }_{\mathrm{pf}} & \text { FGTSVYAYLQEQRLGAAYRLLASGETNVSSAAYRVGYSPAHFSTAFRKRFGVSPKSLR } 300\end{array}$ the FptA structure (Cobessi et al. 2005) indicate that stereospecific siderophore recognition is based on the configuration of the chiral centers $\mathrm{C} 2$ " and $\mathrm{C} 4^{\prime \prime}$ while the configuration at $\mathrm{C}^{\prime}$ seems less important (Hoegy et al. 2009).

A possible stereospecificity of the inner membrane transporters has not been investigated so far but it seems that siderophore translocation across the cytoplasmic membrane is generally less specific than transport through the outer membrane (Köster 2001). It is interesting to note however that the two bacterial species employ transporters belonging to different families, i.e. a single-subunit siderophore transporter for ferriPch uptake in $P$. aeruginosa and a periplasmic protein-dependent $\mathrm{ABC}$ transporter for ferriEPch transport in P. fluorescens.
Stereospecificity in transcriptional regulation with Pch and E-Pch is conferred by the regulatory proteins PchR (Youard and Reimmann 2010). In P. aeruginosa, PchR requires Pch as an effector and no activation occurs with E-Pch (Michel et al. 2005; Youard et al. 2007; Youard and Reimmann 2010). Likewise, the $P$. fluorescens $\mathrm{PchR}$ protein is activated by E-Pch but not by Pch, although the specificity seems somewhat less stringent than that of $P$. aeruginosa PchR (Youard et al. 2007; Youard and Reimmann 2010). How do the PchR homologues distinguish between the two enantiomers? The amino acid sequences at the C-terminal DNA binding domains of both PchR homologues are similar, while their N-terminal domains are less conserved and could thus be involved in siderophore recognition 
(Fig. 5b). Indeed, an analysis of hybrid PchR proteins suggests that an N-terminal domain of about 150 amino acids confers siderophore specificity (Youard and Reimmann 2010).

\section{Concluding remarks and outstanding questions}

The naturally occurring siderophore enantiomer pair Pch and E-Pch offers a unique opportunity to study stereospecificity in iron uptake at several levels. With regard to ferrisiderophore transport across the outer membrane, it will be fascinating to determine the crystal structure of FetA and to compare it with the published structure of the FptA receptor (Cobessi et al. 2005). This will show how siderophore binding pockets have evolved to accommodate ligands of opposite chirality. As mentioned above, in ferriPch and ferriE-Pch translocation across the inner membrane, the importance of siderophore chirality is unknown and it is not clear why mechanistically different transporters are utilized. Swapping the genes for these transporters between $P$. aeruginosa and $P$. fluorescens will allow us to assess a potential stereospecificity in this process.

Regarding siderophore-dependent activation of PchR, there is now good evidence that both siderophores interact, in a stereospecific manner, with the $\mathrm{N}$-terminal part of their cognate PchR protein. In vitro binding studies could confirm this, and, moreover, such experiments could clarify if PchR binds the iron-loaded form of the siderophore as proposed earlier (Michel et al. 2005).

The biosynthetic pathway for E-Pch is not yet fully understood. (i) It is not clear which enzyme epimerizes the second cysteine moiety. A candidate is the methyltransferase domain of PchF, which may have a dual methyltransferase/epimerase function (H.J. Imker and C.T. Walsh, personal communication). (ii) The reductase activity of PchK remains to be demonstrated biochemically. To solve these issues, E-Pch synthesis needs to be reconstituted with purified proteins in vitro, and pathway intermediates and end products need to be identified.

What is the biological role and significance of siderophore enantiomers? Stereochemical variations of siderophores can be regarded as a relatively rare form of defense against siderophore piracy. To our knowledge, the only other known example of siderophore enantiomers is rhizoferrin, which is made as $R, R$-rhizoferrin by the fungus Rhizopus and other members of the Zygomycetes (Drechsel et al. 1992; Thieken and Winkelmann 1992), and as $S, S$-rhizoferrin by the soil bacterium Ralstonia pickettii (Münzinger et al. 1999). Compared with the immense structural variety of pyoverdines made by fluorescent pseudomonads (Meyer 2000; Budzikiewicz 2004), the possibilities of generating diversity through siderophore enantiomers are more limited. Given that secondary siderophores of pseudomonads are often endowed with additional biological activities important in antibiosis, plant defense, inflammation, or biodegradation (Cornelis 2010), it may be that Pch enantiomers could have novel biological activities to be discovered.

Acknowledgments We wish to thank Dieter Haas for critical reading of the manuscript. This work was supported by the Swiss National Science Foundation for Scientific Research (Project 31-113955/1).

\section{References}

Andrews SC, Robinson AK, Rodríguez-Quiñones F (2003) Bacterial iron homeostasis. FEMS Microbiol Rev 27:215-237

Ankenbauer RG, Quan HN (1994) FptA, the Fe(III)-pyochelin receptor of Pseudomonas aeruginosa: a phenolate siderophore receptor homologous to hydroxamate siderophore receptors. J Bacteriol 176:307-319

Ankenbauer RG, Toyokuni T, Staley A, Rinehart KL Jr, Cox CD (1988) Synthesis and biological activity of pyochelin, a siderophore of Pseudomonas aeruginosa. J Bacteriol 170:5344-5351

Bodilis J, Cornelis P (2009) A survey of TonB-dependent receptors in fluorescent pseudomonads. Environ Microbiol Rep 1:256-262

Braud A, Hannauer M, Mislin GLA, Schalk IJ (2009) The Pseudomonas aeruginosa pyochelin-iron uptake pathway and its metal specificity. J Bacteriol 191:3517-3525

Budzikiewicz H (2004) Siderophores of the Pseudomonadaceae sensu stricto (fluorescent and non-fluorescent Pseudomonas spp.). Fortschr Chem Org Naturst 87:81-237

Castignetti D (1997) Probing of Pseudomonas aeruginosa, Pseudomonas aureofaciens, Burkholderia (Pseudomonas) cepacia, Pseudomonas fluorescens, and Pseudomonas putida with the ferripyochelin receptor A gene and the synthesis of pyochelin in Pseudomonas aureofaciens, Pseudomonas fluorescens, and Pseudomonas putida. Curr Microbiol 34:250-257

Cobessi D, Celia H, Pattus F (2005) Crystal structure at high resolution of ferric-pyochelin and its membrane receptor FptA from Pseudomonas aeruginosa. J Mol Biol 352:893-904 
Cornelis P (2010) Iron uptake and metabolism in pseudomonads. Appl Microbiol Biotechnol 86:1637-1645

Cox CD (1980) Iron uptake with ferripyochelin and ferric citrate by Pseudomonas aeruginosa. J Bacteriol 142:581587

Cox CD, Graham R (1979) Isolation of an iron-binding compound from Pseudomonas aeruginosa. J Bacteriol 137: 357-364

Cox CD, Rinehart KL, Moore ML, Cook JC (1981) Pyochelin: novel structure of an iron-chelating growth promoter for Pseudomonas aeruginosa. Proc Natl Acad Sci USA 78:4256-4260

DeClue MS, Baldridge KK, Künzler DE, Kast P, Hilvert D (2005) Isochorismate pyruvate lyase: a pericyclic reaction mechanism? J Am Chem Soc 127:15002-15003

Drechsel H, Jung G, Winkelmann G (1992) Stereochemical characterization of rhizoferrin and identification of its dehydration products. Biometals 5:141-148

Escolar L, Perez-Martin J, de Lorenzo V (1999) Opening the iron box: transcriptional metalloregulation by the Fur protein. J Bacteriol 181:6223-6229

Gaille C, Kast P, Haas D (2002) Salicylate biosynthesis in Pseudomonas aeruginosa. J Biol Chem 277:21768-21775

Gaille C, Reimmann C, Haas D (2003) Isochorismate synthase (PchA), the first and rate-limiting enzyme in salicylate biosynthesis of Pseudomonas aeruginosa. J Biol Chem 278:16893-16898

Guerinot ML (1994) Microbioal iron transport. Annu Rev Microbiol 48:743-772

Harrison AJ, Yu M, Gårdenborg T, Middleditch M, Ramsay RJ, Baker EN, Lott JS (2006) The structure of MbtI from Mycobacterium tuberculosis, the first enzyme in the biosynthesis of the siderophore mycobactin, reveals it to be a salicylate synthase. J Bacteriol 188:6081-6091

Hayen H, Volmer DA (2006) Different iron-chelating properties of pyochelin diastereoisomers revealed by LC/MS. Anal Bioanal Chem 385:606-611

Heinrichs DE, Poole K (1993) Cloning and sequence analysis of a gene $(p c h R)$ encoding an AraC family activator of pyochelin and ferripyochelin receptor synthesis in Pseudomonas aeruginosa. J Bacteriol 175:5882-5889

Heinrichs DE, Poole K (1996) PchR, a regulator of ferripyochelin receptor gene (fptA) expression in Pseudomonas aeruginosa, functions both as an activator and a repressor. J Bacteriol 178:2586-2592

Hoegy F, Lee X, Noel S, Rognan D, Mislin GL, Reimmann C, Schalk IJ (2009) Stereospecificity of the siderophore pyochelin outer membrane transporters in fluorescent pseudomonads. J Biol Chem 284:14949-14957

Ino A, Murabayashi A (2001) Synthetic studies of thiazoline and thiazolidine-containing natural products. Part 3: total synthesis and absolute configuration of the siderophore yersiniabactin. Tetrahedron 57:1897-1902

Kerbarh O, Ciulli A, Howard NI, Abell C (2005) Salicylate biosynthesis: overexpression, purification, and characterization of Irp9, a bifunctional salicylate synthase from Yersinia enterocolitica. J Bacteriol 187:5061-5066

Kerbarh O, Chirgadze DY, Blundell TL, Abell C (2006) Crystal structures of Yersinia enterocolitica salicylate synthase and its complex with the reaction products salicylate and pyruvate. J Mol Biol 357:524-534
Klumpp C, Burger A, Mislin GL, Abdallah MA (2005) From a total synthesis of cepabactin and its 3:1 ferric complex to the isolation of a 1:1:1 mixed complex between iron(III), cepabactin and pyochelin. Bioorg Med Chem Lett 15: $1721-1724$

Köster W (2001) ABC transporter-mediated uptake of iron, siderophores, heme and vitamin B12. Res Microbiol 152:291-301

Künzler DE, Sasso S, Gamper M, Hilvert D, Kast P (2005) Mechanistic insights into the isochorismate pyruvatelyase activity of the catalytically promiscuous PchB from combinatorial mutagenesis and selection. J Biol Chem 280:32827-32834

Liu PV, Shokrani F (1978) Biological activities of pyochelins: iron-chelating agents of Pseudomonas aeruginosa. Infect Immun 22:878-890

Meyer J-M (2000) Pyoverdines: pigments, siderophores and potential taxonomic markers of fluorescent Pseudomonas species. Arch Microbiol 174:135-142

Michel L, Gonzalez N, Jagdeep S, Nguyen-Ngoc T, Reimmann C (2005) PchR-box recognition by the AraC-type regulator PchR of Pseudomonas aeruginosa requires the siderophore pyochelin as an effector. Mol Microbiol 58:495-509

Michel L, Bachelard A, Reimmann C (2007) Ferripyochelin uptake genes are involved in pyochelin-mediated signalling in Pseudomonas aeruginosa. Microbiology 153: $1508-1518$

Münzinger M, Taraz K, Budzikiewicz H, Drechsel H, Heymann P, Winkelmann G, Meyer J-M (1999) S,S-rhizoferrin (enantio-rhizoferrin) - a siderophore of Ralstonia (Pseudomonas) pickettii DSM 6297-the optical antipode of $R, R$-rhizoferrin isolated from fungi. Biometals 12 : 189-193

Namiranian S, Richardson DJ, Russell DA, Sodeau JR (1997) Excited state properties of the siderophore pyochelin and its complex with zinc ions. Photochem Photobiol 65: $777-782$

Ó Cuív P, Clarke P, Lynch D, O’Connell M (2004) Identification of $r h t X$ and fptX, novel genes encoding proteins that show homology and function in the utilization of the siderophores rhizobactin 1021 by Sinorhizobium meliloti and pyochelin by Pseudomonas aeruginosa, respectively. J Bacteriol 186:2996-3005

Ochsner UA, Vasil AI, Vasil ML (1995) Role of the ferric uptake regulator of Pseudomonas aeruginosa in the regulation of siderophores and exotoxin A expression: purification and activity on iron-regulated promoters. J Bacteriol 177:7194-7201

Patel HM, Walsh CT (2001) In vitro reconstitution of the Pseudomonas aeruginosa nonribosomal peptide synthesis of pyochelin: characterization of backbone tailoring thiazoline reductase and $\mathrm{N}$-methyltransferase activities. Biochemistry 40:9023-9031

Patel HM, Tao J, Walsh CT (2003) Epimerization of an Lcysteinyl to a D-cysteinyl residue during thiazoline ring formation in siderophore chain elongation by pyochelin synthetase from Pseudomonas aeruginosa. Biochemistry 42:10514-10527

Paulsen IT, Press CM, Ravel J, Kobayashi DY, Myers GSA et al (2005) Complete genome sequence of the plant 
commensal Pseudomonas fluorescens Pf-5. Nat Biotechnol 23:873-878

Pelludat C, Brem D, Heesemann J (2003) Irp9, encoded by the high-pathogenicity island of Yersinia enterocolitica, is able to convert chorismate into salicylate, the precursor of the siderophore yersiniabactin. J Bacteriol 185:5648-5653

Quadri LEN, Keating TA, Patel HM, Walsh CT (1999) Assembly of the Pseudomonas aeruginosa nonribosomal peptide siderophore pyochelin: in vitro reconstitution of aryl-4,2-bisthiazoline synthetase activity from PchD, PchE and PchF. Biochemistry 38:14941-14954

Reimmann C, Serino L, Beyeler M, Haas D (1998) Dihydroaeruginoic acid synthetase and pyochelin synthetase, products of the $p c h E F$ genes are induced by extracellular pyochelin in Pseudomonas aeruginosa. Microbiology 144:3135-3148

Reimmann C, Patel HM, Serino L, Barone M, Walsh CT, Haas D (2001) Essential PchG-dependent reduction in pyochelin biosynthesis of Pseudomonas aeruginosa. J Bacteriol 183:813-820

Reimmann C, Patel HM, Walsh CT, Haas D (2004) PchC thioesterase optimizes nonribosomal biosynthesis of the peptide siderophore pyochelin in Pseudomonas aeruginosa. J Bacteriol 186:6367-6373

Rinehart KL, Staley AL, Wilson SR, Ankenbauer RG, Cox CD (1995) Stereochemical assignment of the pyochelins. J Org Chem 60:2786-2791

Schlegel K, Taraz K, Budzikiewicz H (2004) The stereoisomers of pyochelin, a siderophore of Pseudomonas aeruginosa. Biometals 17:409-414

Schlegel K, Lex J, Taraz K, Budzikiewicz (2006) The X-ray structure of the pyochelin $\mathrm{Fe}^{3+}$ complex. Z Naturforsch 61c:263-266

Schmidli-Sacherer P, Keel C, Défago G (1997) The global regulator GacA of Pseudomonas fluorescens CHA0 is required for suppression of root diseases in dicotyledons but not in Gramineae. Plant Pathol 46:80-90

Serino L, Reimmann C, Baur H, Beyeler M, Visca P, Haas D (1995) Structural genes for salicylate biosynthesis from chorismate in Pseudomonas aeruginosa. Mol Gen Genet 249:217-228
Serino L, Reimmann C, Visca P, Beyeler M, Della Chiesa V, Haas D (1997) Biosynthesis of pyochelin and dihydroaeruginoic acid requires the iron-regulated $p c h D C B A$ operon in Pseudomonas aeruginosa. J Bacteriol 179:248-257

Stover CK, Pham XQ, Erwin AL, Mizoguchi SD, Warrener P et al (2000) Complete genome sequence of Pseudomonas aeruginosa PAO1, an opportunistic pathogen. Nature 406: 959-964

Terano H, Nomoto K, Takase S (2002) Siderophore production and induction of iron-regulated proteins by a microorganism from rhizosphere of barley. Biosci Biotechnol Biochem 66:2471-2473

Thieken A, Winkelmann G (1992) Rhizoferrin: a complexone type siderophore of the Mucorales and Entomophthorales (Zygomycetes). FEMS Microbiol Lett 94:37-42

Thomas MS (2007) Iron acquisition mechanisms of the Burkholderia cepacia complex. Biometals 20:431-452

Tseng C-F, Burger A, Mislin GLA, Schalk IJ, Yu SS-F, Cahn SI, Abdallah MA (2006) Bacterial siderophores: the solution stoichiometry and coordination of the $\mathrm{Fe}$ (III) complexes of pyochelin and related compounds. J Biol Inorg Chem 11:419-432

Visca P, Colotti G, Serino L, Verzili D, Orsi N, Chiancone E (1992) Metal regulation of siderophore synthesis in Pseudomonas aeruginosa and functional effects of siderophore-metal complexes. Appl Environ Microbiol 58: 2886-2893

Wandersman C, Delepelaire P (2004) Bacterial iron sources: from siderophores to hemophores. Annu Rev Microbiol 58:611-647

Youard ZA, Reimmann C (2010) Stereospecific recognition of pyochelin and enantio-pyochelin by the PchR proteins in fluorescent pseudomonads. Microbiology 156:1772-1782

Youard ZA, Mislin GLA, Majcherczyk PA, Schalk IJ, Reimmann C (2007) Pseudomonas fluorescens CHA0 produces enantio-pyochelin, the optical antipode of the Pseudomonas aeruginosa siderophore pyochelin. J Biol Chem 282:35546-35553

Zaitseva J, Lu J, Olechoski KL, Lamb AL (2006) Two crystal structures of the isochorismate pyruvate-lyase from Pseudomonas aeruginosa. J Biol Chem 281:33441-33449 